\title{
Findings from the first round of the National Audit of Care at the End of Life (NACEL)
}

\author{
Authors: Suzanne Kite, ${ }^{A}$ Elizabeth Rees, ${ }^{A}$ Claire Holditch ${ }^{A}$ and Debbie Hibbert ${ }^{A}$
}

\section{Introduction}

NACEL is a national comparative audit of the quality and outcomes of care experienced by the dying person and those important to them during the last admission leading to death in acute, community hospitals and mental health inpatient providers in England and Wales.

\section{Materials and methods}

The audit, first undertaken during 2018/19, comprised:

$>$ an organisational-level audit covering trusts (in England) and health boards (in Wales)

$>$ a case note review completed by acute and community providers only, reviewing all deaths in April 2018 (acute providers) or deaths in April-June 2018 (community providers)

> a quality survey completed online, or by telephone, by the bereaved person.

Data were collected between June and October 2018. 206 trusts in England and 8 Welsh organisations took part in at least one element of the audit ( $97 \%$ of eligible organisations). A total of 11,034 case note reviews were included.

\section{Results and discussion}

Documentation that a person may die imminently was high. For half of patients, imminent death was recognised less than one and a half days before they died, leaving a limited amount of time to discuss and implement an individual plan of care.

People's experience of care was good, excellent or outstanding in most cases ( $80 \%)$, as reported by the quality survey. However, $20 \%$ felt that there was scope to improve the quality of care and sensitive communication with both the patient and the family/ others.

Governance of end-of-life care was strong.

Improvement is required in the documentation of an individual plan of care (there was documented evidence of a plan for $62 \%$ of people who died). Similarly, for one-third of people who died, a discussion about the plan of care, and discussions about medication, hydration and nutrition, had not been recorded.

\section{Conclusion}

The full audit findings from the first round were published on 11 July 2019. ${ }^{1}$

The second round of NACEL is running in 2019/2020 and data collection is currently underway, involving acute and community hospital providers. Mental health providers are not participating in round 2 of NACEL.

\section{Conflicts of interest}

None declared.

\section{Reference}

1 Healthcare Quality Improvement Partnership. The National Audit of Care at the End of Life: First round of the audit (2018/19) report for England and Wales. HQIP, 2019. 